\title{
Penile verrucous squamous cell carcinoma: A rare case report
}

\author{
Omer Yuksel, Emre Karabay, Osman Bilen, Çağatay Tosun, Levent Verim \\ Haydarpasa Numune Training and Research Hospital, University of Health Sciences, Department of Urology, \\ Uskudar/Istanbul Turkey.
}

\begin{abstract}
Summary Penile cancer is a rare type of urological cancer. Predisposing factors include phimosis, poor hygiene, and smoking. Circumcision in early childhood has been shown to be protective against penile cancer. About $95 \%$ of penile cancers are squamous cell carcinomas, while verrucous type is a rare variant with frequent recurrences, but with a favorable prognosis. The majority of patients are asymptomatic; however, patients may present with pain, discharge, and bad odor depending on the severity of the disease. Although hospital admission is often late due to psychosocial factors, cancer is often localized. Herein, we report a 61-year-old circumcised patient presenting with painful penile mass who was diagnosed with a penile verrucous squamous cell carcinoma in the light of literature data.
\end{abstract}

KEY WORDS: Penile cancer; Verrucous cancer; Penectomy.

Submitted 17 November 2019; Accepted 12 December 2019

\section{INTRODUCTION}

Penile cancer is the rarest of urological malignancies in male. Its annual incidence is $1 / 100,000$ in the United States (US) and Europe. As it is more common in many countries worldwide, it is considered a global health problem (1). According to the American Cancer Society, 2,080 newly diagnosed penile cancer patients and 410 penile cancer-related death have been estimated in the US in 2019 (2). Its incidence is the highest in Brazil, Uganda, and India and the lowest in the Jewish and Muslim communities in which male infants and children are mostly circumcised. Male circumcision in early childhood has been shown to reduce the risk for penile cancer by three to five-fold, probably as it prevents chronic irritation (3). Predisposing factors being uncircumcised, balanoposthitis, balanitis xerotica obliterans (BXO), ultraviolet phototherapy, sexual promiscuity, sexual intercourse in early adolescence, history of condyloma, tobacco smoking, and sexual intercourse with a partner infected with Human Papillomavirus (HPV) (4). In addition, a high-risk HPV-DNA positivity has been associated with reduced survival (5).

The most common type of penile cancer is squamous cell carcinoma (SCC). Penile SCC can be divided into several subtypes. The most common subtypes include usual SCC (48 to $65 \%$ ), basaloid carcinoma (4 to $10 \%$ ), warty carcinoma ( 7 to $10 \%$ ), verrucous carcinoma (3 to $8 \%$ ), papillary carcinoma (5 to 15\%), and mixed carcinomas (9 to $10 \%$ ). Verrucous carcinoma is a rare variant of well- differentiated SCC with low malignancy potential. Premalignant lesions include penile cutaneous horn, Bowenoid papulosis, and BXO, while potential risk factors for the development of penile cancer are penile intraepithelial neoplasms, erythroplasia of Queyrat, and Bowen disease (3). Of these lesions, about 30\% result in invasive cancer.

Penile cancer, which is mostly seen in individuals aged 50 to 70 years, affects glans of penis (48\%), prepuce (21\%), both glans and prepuce (15\%), coronal sulcus $(6 \%)$, and penile shaft $(<2 \%)$. Initial physical examination manifestations may widely vary from a small redness to a large ulcer or infiltrative lesion. Patients may present with itching, pain, bleeding, discharge, and bad odor depending on the severity of the disease. Hospital admission is often late due to psychosocial factors; 15 to $60 \%$ of patients seek a medical diagnosis and treatment at least one year after the symptom onset. Nonetheless, the disease is localized in $66 \%$ of patients. In the initial admission, inguinal lymph nodes must be evaluated. Penile cancer metastasizes in a predictable pattern with superficial and deep inguinal lymph nodes occurring first, followed by pelvic and periaortic lymph nodes. Distant metastasis is very rare (1 to 10\%) and mostly occurs in the late stages of the disease (6). Disease staging is based on the American Joint Committee on Cancer TNM Staging System depending on the depth of invasion, lymph node invasion, and distant metastases $(6,7)$. Herein, we report a 61-year-old circumcised patient presenting with painful penile mass who was diagnosed with a penile verrucous SCC in the light of literature data.

\section{Case report}

A 61-year-old male patient who was circumcised at the age of 13 years was admitted to our outpatient clinic with a painful penile mass. Although his complaints were present for two years, he did not seek a medical treatment due to psychosocial factors. Upon increased penile pain, he applied to our clinic. His medical history revealed no smoking history, except being a social smoker, dysuria, having multiple sexual partners or sexually transmitted disease, or previous surgery. He was on regular medical treatment for hypertension and diabetes mellitus. His familial medical history revealed rectal cancer in his father. Physical examination revealed a $2-\mathrm{cm}$ 


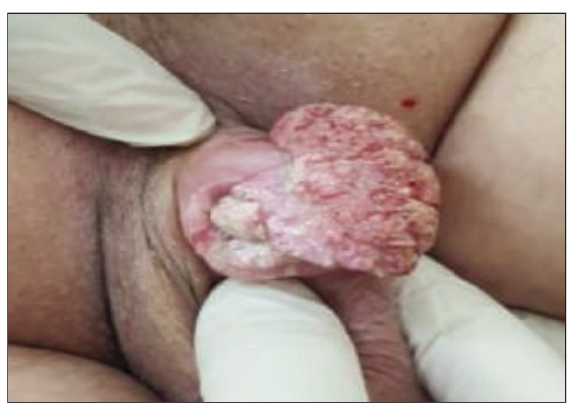

Figure 1.

Penile mass on physical examination.

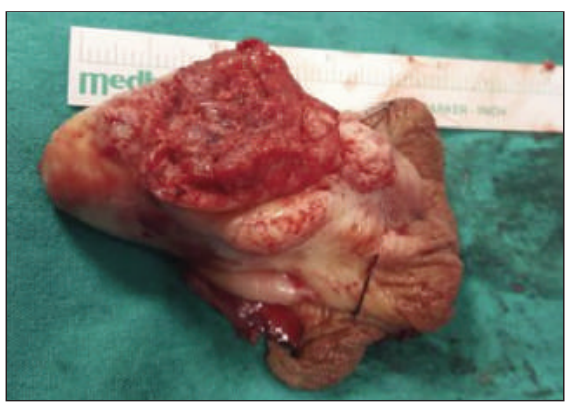

Figure 2.

Partial penectomy material.

ulcerative mass with bleeding and itching advancing toward the coronal sulcus from the dorsal layer of the glans without urethral meatus involvement (Figure 1). Poor penile hygiene was observed. Inguinal region examination showed no palpable lymphadenopathy. Skin biopsy of the penile ulcer was performed. Pathological examination result reported a well-differentiated penile verrucous SCC. Magnetic resonance imaging and positron emission tomography-computed tomography revealed TaNoMo clinical stage and partial penectomy was performed (Figure 2). Partial penectomy pathology was reported T3NxMx. No recurrence or metastasis was observed in the postoperative six months of follow-up.

\section{Discussion}

Penile cancer is a rare type of cancer which accounts for less than $1 \%$ of cancers in men (8). Its prevalence is higher in developing countries. It more frequently affects uncircumcised, white men with low income (9). Squamous cell carcinoma is the most common type and $33 \%$ of SCCs originate from premalignant lesions. Possible mechanisms which stimulate malignant transformation include chronic irritation, tobacco smoking, and poor penile hygiene which induce chronic inflammation, metaplasia, and differentiation (10). Certain types of viral infections such as HPV may also lead to penile cancer through DNA damage. In addition, damages in tumor suppressor genes such as p53 disrupt the cell cycle, resulting in DNA damage and malignant transformation $(4,5)$.

Verrucous SCC of the oral cavity was first defined by Ackerman in 1948. It accounts for 3 to $8 \%$ of penile cancers and $20 \%$ of verruciform lesions. It is a rare variant of exophytic, papillomatous, low-grade, and well-differentiated SCC. As verrucous SCCs mostly present with squamous epithelial hyperplasia and keratinization, misdiagnosis is common, when diagnostic biopsy fails.
Therefore, biopsy is strongly recommended for definitive diagnosis. Its etiology has not been fully understood, yet (11).

Earlier studies have demonstrated that verrucous SCCs are associated with low-risk HPV infection (12). However, later studies using broad-spectrum HPV polymerase chain reaction testing have revealed controversial results. In a study, Rubin et al. (12) reported that basaloid and warty SCCs were associated with HPV, while the HPV positivity rate was $33.3 \%$ and $34.9 \%$ for verrucous and usual SCCs, respectively. In another study, Stankiewicz et al. (13) reported an HPV-DNA positivity of $23 \%$ and $59 \%$ for verrucous and usual SCCs, respectively. These findings indicate a low-degree association between penile verrucous SCC and HPV.

Although most of the current data regarding verrucous SCCs are based on case reports and case series, surgery is the mainstay of the treatment. The main strategy is wide excision of the mass or partial penectomy. Radical penectomy can be performed in rare cases. Recurrence of penile verrucous SCCs is high; one case with recurrence after 30 years has been reported in the literature (14). In early recurrence, additional resection and even radical penectomy can be performed.

Distant metastasis is extremely rare and is not seen in almost none of patients with penile verrucous SCC. Therefore, inguinal lymphadenectomy is reserved for only very few patients. Hatzichristou et al. (15) performed inguinal lymphadenectomy in selected patients with penile verrucous SCC; however, no specific lesion could be detected. Thus, prophylactic inguinal lymphadenectomy is not recommended for this patient population. Early diagnosis and prevention are of utmost importance for the management of penile cancer. Neonatal circumcision, smoking cessation, and HPV vaccination have been suggested to decrease the incidence of penile cancer.

\section{RefERENCES}

1. Parkin DM, Whelan SL, Ferlay J, et al. Cancer Incidence in Five Continents. Vol. VIII. http://www.iarc.fr/en/Publicaions/PDFsonline/Cancer-Epidemiology/IARC-Scientific Publication-No.155.Accessed March 12,2014.

2. American Cancer Society. Cancer Facts \& Figures 2019. Atlanta: American Cancer Society; 2019.

3. Barnholtz-Sloan JS, Maldonado JL, Pow-Sang J, Guliano AR. Incidence trends in primary malignant penile cancer. Urol Oncol. 2007; 25:361-367.

4. Pizzocaro G, Algaba F, Horenblas S, et al. EAU penile cancer guidelines 2009. Eur Urol. 2010; 57:1002-1012.

5. Bezerra AL, Lopes A, Santiago GH, et al. Human papillomavirus as a prognostic factor in carcinoma of the penis: analysis of 82 patients treated with amputation and bilateral lymphadenectomy. Cancer. 2001; 91:2315-2321.

6. Marchionne E, Perez C, Hui A, Khachemoune A. Penile squamous cell carcinoma: a review of the literature and case report treated with Mohs micrographic surgery. An Bras Dermatol. 2017; 92:95-99.

7. Edge SB, Byrd DR, Compton CC, et al. AJCC Cancer Staging Manuel. (7th edn), New York, NY: Springer. 2010.

8. Wilson CN, Sathiyasusuman A. Associated risk factors of STIs 
and multipl sexual relationships among youths in Malawi. PLoS One. 2015; 10:e0134286.

9. Morrison BF. Risk factors and prevalence of penile cancer. West Indian Medical Journal. 2014; 63:559-560.

10. Velazquez EF, Cubilla AL. Lichen sclerosus in 68 patients with squmous cell carcinoma of the penis: frequent atypias and correlation with special carcinoma variants suggest a precancerous role. Am Surg Patol. 2003; 27:1448-1453.

11. Schwartz RA. Verrucous carcinoma of the skin and the mucosa. J Am Acad Dermatol. 1995; 32:1-21.

12. Rubin MA, Kleter B, Zhou M, et al. Detection and typing of human papillomavirus DNA in penile carcinoma: evidence for mul- tiple independent pathways of penile carcinogenesis. Am J Pathol. 2001; 159:1211-1218

13. Stankiewicz E, Kudahetti SC, Proxse DM, et al. HPV infection and immunochemical detection of cell-cycle markers in verrucous carcinoma of the penis. Mod Pathol. 2009; 22:1160-1168.

14. Alouani I, Barki A, Zizi N, Dikhaye S. Penile verrucous carcinoma: a new case report in a circumcised man. Clin Oncol. 2019; 4:1594.

15. Hatzichristou DG, Apostolidis A, Tzortzis V, et al. Glansectomy: an alternative surgical treatment for Buschke-Löwenstein tumors of the penis. Urology 2001; 57:966-969.

\section{Correspondence}

Omer Yuksel, MD

dr_omer_yuksel@hotmail.com

Emre Karabay, MD (Corresponding Author)

emrekarabay@gmail.com

Osman Bilen, MD

osmanbilen1212@gmail.com

Çağatay Tosun, $M D$

cagataytosun@hotmail.com

Levent Verim, MD

leventverim@hotmail.com

Haydarpasa Numune Training and Research Hospital, University of Health Sciences, Dept. Urology

Tibbiye Street. No: 2334668 Uskudar/Istanbul (Turkey) 